\title{
RR Lyrae variables in the globular cluster M 5
}

\author{
J. Kaluzny ${ }^{1}$, A. Olech ${ }^{2}$, I. Thompson ${ }^{3}$, W. Pych ${ }^{2}$, W. Krzeminski ${ }^{1,3}$, and A. Schwarzenberg-Czerny ${ }^{1,4}$ \\ 1 Copernicus Astronomical Center, ul. Bartycka 18, 00-716 Warsaw, Poland \\ e-mail: jka,wk,alex@camk.edu.pl \\ 2 Warsaw University Observatory, Al. Ujazdowskie 4, 00-478 Warsaw, Poland \\ e-mail: olech,pych@sirius.astrouw.edu.pl \\ 3 Carnegie Institution of Washington, 813 Santa Barbara Street, Pasadena, CA 91101, U.S.A. \\ e-mail: ian@ociw.edu \\ 4 Astronomical Observatory of Adam Mickiewicz University, ul. Sloneczna 36, 61-286 Poznan, Poland
}

Received July 2, 1999; accepted January 27, 2000

\begin{abstract}
We present $V$-band CCD photometry of $65 \mathrm{RR}$ Lyr variables from the globular cluster M 5. We have estimated the basic physical parameters for 16 RRc stars and 26 RRab stars using a Fourier decomposition of the light curves of the variables. The mean values of mass, luminosity, effective temperature and relative helium abundance for the RRc stars are measured to be $\mathcal{M}=0.54 \mathcal{M}_{\odot}$, $\log \left(L / L_{\odot}\right)=1.69, T_{\text {eff }}=7353 \mathrm{~K}$ and $Y=0.28$, respectively. For the RRab variables the derived mean values of absolute magnitude, metallicity and effective temperature are: $M_{V}=0.81,[\mathrm{Fe} / \mathrm{H}]=-1.23$ and $T_{\text {eff }}=6465 \mathrm{~K}$. We find that the $V$ amplitude of an RRab star for a given period is a function of metal abundance rather than Oosterhoff type.

We find significant problems with the calibration of both the zero point and the scale of the luminosities measured with the Fourier technique. The apparent distance modulus derived from RRc stars is equal to $14.47 \pm 0.11$ and it is in good agreement with recent determinations. On the other hand distance modulus obtained from the sample of RRab stars (calibrated by the Baade-Wesselink observations of field RR Lyr variables) is significantly smaller and equal to $14.27 \pm 0.04$
\end{abstract}

Key words: stars: RR Lyr — stars: variables — globular clusters: individual: M 5

\section{Introduction}

NGC $5904=\mathrm{M} 5=\mathrm{C} 1516+022$ is one of the richest globular clusters in the northern hemisphere. It is placed only

Send offprint requests to: A. Olech, e-mail: olech@sirius.astrouw.edu.pl
$7 \mathrm{kpc}$ from the Sun, $5.5 \mathrm{kpc}$ from the Galactic Center and $4.9 \mathrm{kpc}$ above the plane of the Galactic disc (Zinn 1985), and thus has low reddening, $E(B-V)=0.03$ (Harris 1996). The above properties make M 5 an excellent target for detailed studies, especially in the field of variable stars.

Spectroscopic investigations of M 5 yield metallicity determinations varying from $[\mathrm{Fe} / \mathrm{H}]=-1.0$ (Butler 1975) to $[\mathrm{Fe} / \mathrm{H}]=-1.4$ (Zinn 1985). Carretta \& Gratton (1997) obtained $[\mathrm{Fe} / \mathrm{H}]=-1.11 \pm 0.03$ on their newly introduced scale of GC metallicities.

M 5 is known to contain more than 140 RR Lyr variables (Clement 1997; Sandquist et al. 1996; Caputo et al. 1999). CCD VI photometry for 49 of them was recently presented by Reid (1996) who gives references to earlier papers dealing with RR Lyr stars in M 5. Recently, Kaluzny et al. (1999) reported the discovery of 5 faint variables in the cluster field: four SX Phe stars and one eclipsing binary. They also showed that several candidate eclipsing binaries identified by Reid (1996) and Yan \& Reid (1996) are non-variable objects. Drissen \& Shara (1998) used HST images to look for variable stars in the core of M 5. They identified one variable blue straggler, probably an eclipsing binary, and several new RR Lyr stars. New $B V$ photometry of the M 5 RR Lyr variables was also reported by Caputo et al. (1999). And recently Olech et al. (1999b) using newly developed image subtraction method discovered four new variables in this cluster.

In this paper we present results based on time-series photometry of RR Lyr stars in M 5 obtained in 1997 at Las Campanas Observatory. The detailed description of the observational runs and reduction process were published elsewhere (Kaluzny et al. 1999). In short, the observing material consisted of $275 \mathrm{~V}$-band frames collected during the period from May 9 through August 13 1997. Most of the data were taken during a single sub-run in May 1997. 
Table 1. Light curve parameters for RRc variables from M 5

\begin{tabular}{|c|c|c|c|c|c|c|c|c|c|c|c|c|}
\hline \hline Star & Period & $A_{V}$ & $A_{0}$ & $A_{1}$ & $R_{21}$ & $\sigma_{R_{21}}$ & $\phi_{21}$ & $\sigma_{\phi_{21}}$ & $\phi_{31}$ & $\sigma_{\phi_{31}}$ & $\phi_{41}$ & $\sigma_{\phi_{41}}$ \\
\hline \hline V15 & 0.336776 & 0.41 & 15.064 & 0.206 & 0.087 & 0.010 & 3.067 & 0.117 & 7.130 & 0.142 & 3.571 & 0.243 \\
V31 & 0.300575 & 0.50 & 15.069 & 0.261 & 0.157 & 0.004 & 3.100 & 0.019 & 6.117 & 0.028 & 3.356 & 0.042 \\
V35 & 0.308177 & 0.46 & 14.967 & 0.234 & 0.154 & 0.004 & 3.431 & 0.045 & 6.542 & 0.073 & 3.644 & 0.106 \\
V40 & 0.317334 & 0.43 & 15.051 & 0.220 & 0.114 & 0.005 & 3.147 & 0.034 & 6.549 & 0.046 & 3.975 & 0.091 \\
V44 & 0.329576 & 0.42 & 15.027 & 0.214 & 0.107 & 0.009 & 2.899 & 0.080 & 6.967 & 0.128 & 4.026 & 0.259 \\
V55 & 0.328938 & 0.41 & 15.086 & 0.210 & 0.119 & 0.010 & 3.438 & 0.080 & 7.132 & 0.133 & 4.024 & 0.188 \\
V57 & 0.284673 & 0.49 & 15.050 & 0.246 & 0.179 & 0.004 & 3.305 & 0.032 & 6.065 & 0.065 & 3.256 & 0.076 \\
V62 & 0.281412 & 0.53 & 15.099 & 0.262 & 0.187 & 0.004 & 3.107 & 0.030 & 5.808 & 0.067 & 3.036 & 0.079 \\
V66 & 0.350658 & 0.44 & 15.032 & 0.225 & 0.053 & 0.005 & 3.299 & 0.122 & 7.343 & 0.095 & 4.113 & 0.216 \\
V73 & 0.340111 & 0.51 & 14.974 & 0.262 & 0.095 & 0.008 & 3.364 & 0.085 & 7.047 & 0.098 & 3.854 & 0.150 \\
V76 & 0.432544 & 0.40 & 14.828 & 0.192 & 0.036 & 0.005 & 5.524 & 0.201 & 8.276 & 0.109 & 5.315 & 0.212 \\
V78 & 0.264798 & 0.39 & 15.094 & 0.196 & 0.158 & 0.005 & 3.090 & 0.038 & 6.209 & 0.137 & 2.698 & 0.173 \\
V79 & 0.333089 & 0.35 & 14.947 & 0.177 & 0.147 & 0.011 & 3.134 & 0.151 & 7.033 & 0.237 & 3.379 & 0.388 \\
V80 & 0.336549 & 0.39 & 15.042 & 0.196 & 0.117 & 0.005 & 2.998 & 0.057 & 7.194 & 0.116 & 4.014 & 0.146 \\
V88 & 0.328070 & 0.43 & 15.034 & 0.220 & 0.123 & 0.014 & 3.496 & 0.100 & 6.626 & 0.183 & 4.045 & 0.285 \\
V130 & 0.326624 & 0.42 & 14.882 & 0.212 & 0.033 & 0.028 & 4.460 & 0.876 & 7.544 & 0.395 & 4.464 & 0.776 \\
\hline Mean & 0.324994 & 0.44 & 15.015 & 0.221 & 0.117 & - & 3.429 & - & 6.849 & - & 3.798 & - \\
\hline \hline
\end{tabular}

Two fields with a significant overlap at the cluster center were monitored.

\section{Results}

Our search for variable stars in M 5 has identified 65 RR Lyrae variables. All these stars were previously known (Sawyer Hogg 1973; Sandquist et al. 1996; Clement 1997). We have detected 49 fundamental mode pulsators (Bailey type RRab), 15 first overtone pulsators (Bailey type RRc), and one possible second mode pulsator (Bailey type RRe). In this paper we use names assigned by Sawyer Hogg (1973) with the exception of V963 (Zhukov 1971).

We fitted our $V$-band light curves with Fourier series of the form:

$V=A_{0}+\sum_{j=1}^{8} A_{j} \cdot \sin \left(j \omega t+\phi_{j}\right)$

where $\omega=2 \pi / P$ and $P$ is the pulsation period of the star. Least squares fits were computed by orthogonal projections onto trigonometric polynomials using a method developed by Schwarzenberg-Czerny (1997) and Schwarzenberg-Czerny \& Kaluzny (1998). In this way we determined the values of $\omega, A_{j}$ and $\phi_{j}$ together with their errors, and we calculated amplitude combinations $R_{j 1}=$ $R_{j} / R_{1}$ and phase differences defined as $\phi_{j 1}=\phi_{j}-j \cdot \phi_{1}$. These quantities will be used in the following analysis.

Using the derived periods we constructed phased $V$ band light curves which are presented in Fig. 1. The periods of the cluster RRc stars range from 0.2648 to 0.4325 days with a mean period of 0.3250 days. The periods of the RRab variables are between 0.4497 and 0.8453 days with a mean value of 0.554 days. These properties place M 5 among the Oosterhoff type I clusters.
In Fig. 2 we compare our photometry with observations given by Reid (1996). We plot $A_{0}-<V>$ (Reid) vs $A_{0}$, where $A_{0}$ is the mean magnitude as measured by the Fourier decomposition of the light curves. Some stars in common to the two samples have not been plotted (V6, V54, and V91: our light curve or Reid's measured with particularly poor signal-to-noise; V24 and V52: Reid's data do not sample the maxima of the light curves). For the remaining 32 measurements in common the mean value of $A_{0}-<V>$ (Reid) $=-0.011 \pm 0.026$.

In Fig. 3 we present the relations between the pulsational period and the peak to peak $A_{V}$ amplitude, $R_{21}$, $\phi_{21}, \phi_{31}$ and $\phi_{41}$. In these plots RRc stars occupy locations quite distinct from RRab stars, and in the following sections we discuss these two groups of stars separately. The relative lack of scatter in the relations presented in Fig. 3 attests to the overall quality of our light curves (cf. Clement et al. 1992; Simon \& Clement 1993).

\subsection{RRc stars}

The resulting Fourier parameters for RRc stars are presented in Table 1. In the following sections we discuss individual RRc stars with atypical light curves, and then the derivation of physical parameters of the RRc stars from the Fourier coefficients.

\subsubsection{Individual stars}

The light curve of V130 reveals scatter which is about 10 times larger than the scatter observed for most variables. However, prewhitening the observations with the base period and its 3 harmonics leaves no power in excess of $0.02 \mathrm{mag}$ amplitude in the frequency range $0-300 \mathrm{c} / \mathrm{d}$. 


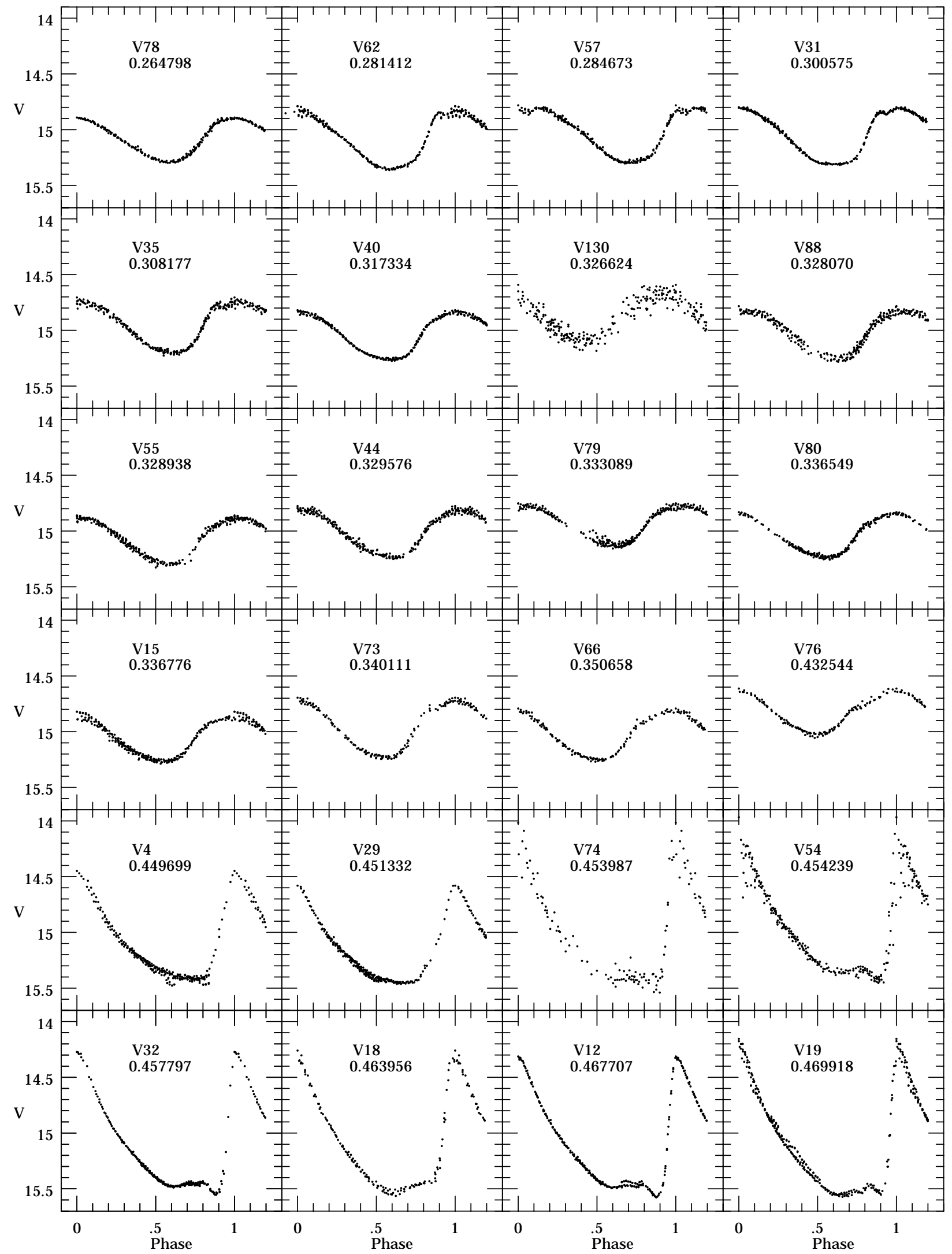

Fig. 1. $V$-band light curves of RR Lyr variables in M 5. The stars are plotted according to the increasing period 


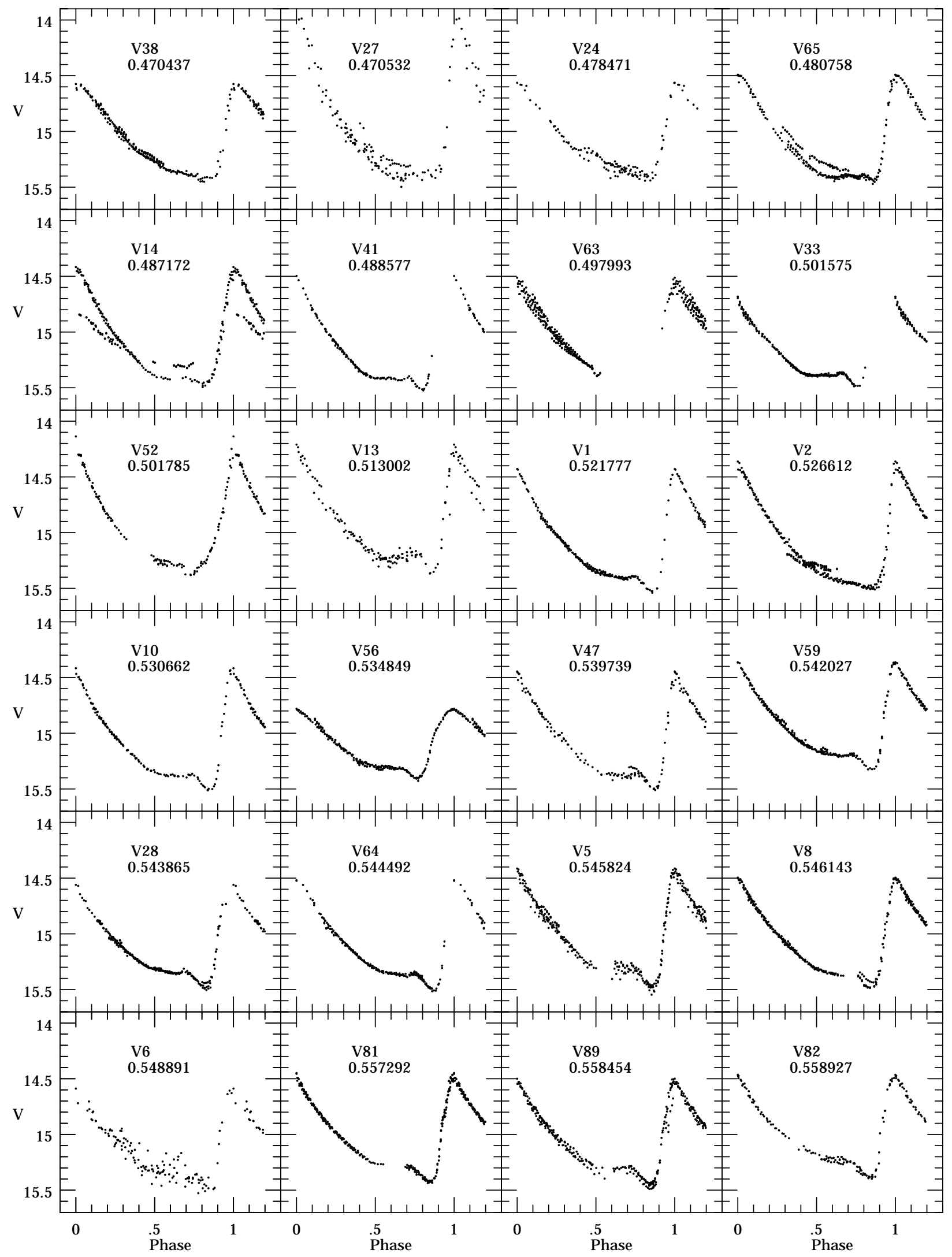

Fig. 1. continued 


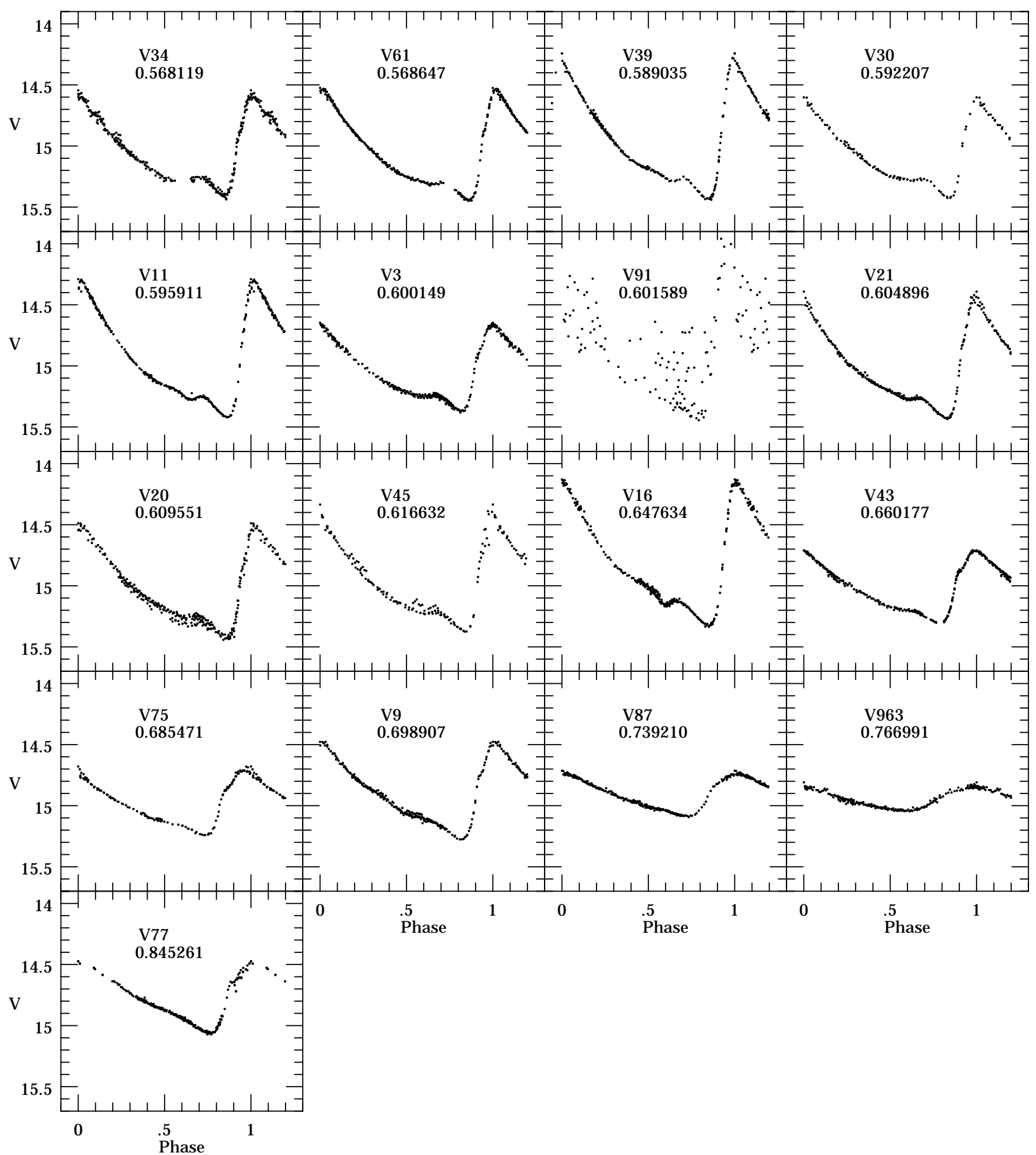

Fig. 1. continued

Inspection of the frames reveals 3 nearby companion stars, and we conclude that the scatter caused by crowding. The shift in the light curve of V88 also appears to be instrumental in origin (our light curves were obtained from two overlapping fields and some stars laying at large distance from the overlapping region may have small shift between light curves obtained from different fields). This star has a frequency $3.05 \mathrm{c} / \mathrm{d}$, so that the phasing of nearby observations is similar. Removal of the base frequency and its harmonics leaves a very low frequency residual signal.

Two stars are outliers in the $A_{V}-\log P, R_{21}-\log P$, $\phi_{21}-\log P$ and $\phi_{31}-\log P$ relations presented in Fig. 3 . One of these is V76 - the RRc variable with the longest period in our sample at $0.4325 \mathrm{~d}$. Another outlying object is V78 - the star with the shortest period in our sample. Recently Minniti et al. (1997) published a study 


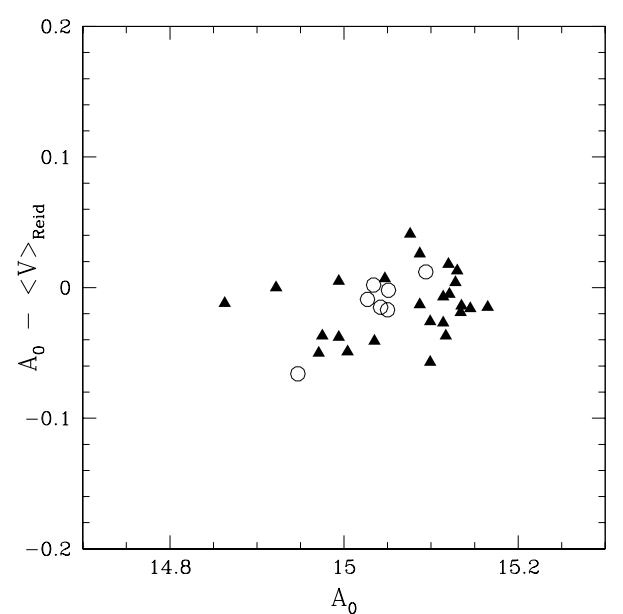

Fig. 2. A comparison of the photometry presented in this work with the observations by Reid (1996). RRc variables are plotted with circles and RRab stars with triangles

of the RR Lyr variables in the MACHO Collaboration database. They found three peaks in the period distribution of stars in the Large Magellanic Cloud and in the Galactic bulge. The two most prominent peaks are the RRab and RRc pulsators and the lowest peak, at a period of about $0.27-0.28 \mathrm{~d}$, was interpreted as due to RRe stars (pulsations in the 2 nd overtone). A similar result for RR Lyr variables from the Galactic bulge was obtained by Olech (1997) and for variables from the globular cluster IC 4499 by Walker \& Nemec (1996). Theoretical calculations performed by Sandage (1981) support the hypothesis that RR Lyr stars with the shortest periods and smallest amplitudes may be RRe type pulsators. Additionally Stellingwerf et al. (1987) predicted that, if RRe stars exist, they should have light curves that have a sharper peak at maximum light than the 1st overtone pulsators. Variable V78 fits this description very well. It has the shortest period among our sample of RR Lyr stars, its amplitude is low and its light curve (see Fig. 1) is more asymmetric than the light curves of other RRc stars.

The light curves of other RRc variables with the shortest periods, from $0.28^{\mathrm{d}}$ to $0.30^{\mathrm{d}}$, i.e. V31, V35, V57 and V62, are remarkably similar both in their general shape and in fine details. It is perhaps interesting that they all display small bumps just before the maxima and that the scatter of observations around the maxima appears to be slightly larger than the scatter at minimum light. Light curves of other stars with periods just above the range $0.28-0.30^{\mathrm{d}}$ do not show such behavior. Because of this consistency, instrumental origin is not a likely cause of these effects (e.g. saturation of the CCD images at maximum light). If confirmed by further observations of comparable accuracy, these effects could reveal potentially interesting dynamical processes. We have already argued that V78, the star with the shortest period in our sample, is likely to pulsate in the second overtone. Hence, one has
Table 2. Physical parameters derived for RRc Lyrae variables from M 5

\begin{tabular}{|r|c|c|c|c|c|r|c|}
\hline \hline Star & Mass & $\sigma_{\text {Mass }}$ & $\log L$ & $\sigma_{\log L}$ & $T_{\text {eff }}$ & $\sigma_{\mathrm{T}}$ & $Y$ \\
\hline \hline V15 & 0.508 & 0.018 & 1.687 & 0.008 & 7338 & 13 & 0.285 \\
V31 & 0.618 & 0.004 & 1.694 & 0.002 & 7363 & 2 & 0.276 \\
V35 & 0.563 & 0.010 & 1.681 & 0.004 & 7377 & 6 & 0.283 \\
V40 & 0.570 & 0.007 & 1.694 & 0.003 & 7346 & 4 & 0.279 \\
V44 & 0.523 & 0.017 & 1.687 & 0.007 & 7345 & 12 & 0.284 \\
V55 & 0.501 & 0.017 & 1.676 & 0.008 & 7363 & 12 & 0.288 \\
V57 & 0.609 & 0.010 & 1.673 & 0.004 & 7416 & 6 & 0.283 \\
V62 & 0.646 & 0.011 & 1.683 & 0.004 & 7404 & 6 & 0.278 \\
V66 & 0.491 & 0.012 & 1.693 & 0.006 & 7315 & 8 & 0.284 \\
V73 & 0.521 & 0.013 & 1.696 & 0.006 & 7320 & 9 & 0.281 \\
V76 & 0.432 & 0.012 & 1.734 & 0.006 & 7182 & 10 & 0.277 \\
V78 & 0.566 & 0.020 & 1.632 & 0.008 & 7509 & 13 & 0.297 \\
V79 & 0.517 & 0.031 & 1.688 & 0.014 & 7340 & 22 & 0.284 \\
V80 & 0.499 & 0.015 & 1.683 & 0.007 & 7345 & 10 & 0.286 \\
V88 & 0.569 & 0.026 & 1.704 & 0.011 & 7318 & 17 & 0.276 \\
V130 & 0.450 & 0.045 & 1.649 & 0.023 & 7410 & 37 & 0.300 \\
\hline \hline
\end{tabular}

to consider a possibility that some sort of interaction between the first and second overtones is responsible for the effects observed in the light curves of V31, V35, V57 and V62.

\subsubsection{Physical parameters}

Simon \& Teays (1982); Simon (1989) and Simon \& Clement (1993) (hereafter SC) have presented a method of estimating the masses, luminosities, effective temperatures and helium abundances of RRc stars based only on the Fourier parameters of $V$-band light curves. The relevant equations are summarized in Olech et al. (1999a). The existence of a relation between the masses, luminosities, temperatures and metallicities of pulsating stars and their Fourier parameters is based on hydrodynamic pulsation models. However, the details of the specific calibrations based on models are still subject to revisions. Hence, we list in Table 1 the cluster averages and standard deviations of the Fourier parameters as model-free characteristics of the pulsating stars in this particular cluster. Please note that the standard deviations refer to the spread of derived parameters and not to observational uncertainties (errors of the Fourier parameters are listed in Table 1).

Table 2 presents the estimated masses, luminosities, effective temperatures and helium abundances for all of the RRc stars in our sample. The errors presented in Table 2 are calculated from the error propagation law. Only two stars from Table 2 have errors of $\phi_{31}$ larger than 0.2 (V79 and V130), and we have omitted them in further analysis.

In Fig. 4 we have plotted the calculated values of $\log \left(L / L_{\odot}\right)$ (hereinafter $\log L$ ) against the observed 
Table 3. Mean parameters for RRc stars in globular clusters after Clement \& Shelton (1997) and Kaluzny et al. (1998)

\begin{tabular}{|l|c|c|c|c|c|c|c|}
\hline \hline Cluster & $\begin{array}{c}\text { Oosterhoff } \\
\text { type }\end{array}$ & {$[\mathrm{Fe} / \mathrm{H}]$} & $\begin{array}{c}\text { No. of } \\
\text { stars }\end{array}$ & $\begin{array}{c}\text { mean } \\
\text { mass }\end{array}$ & $\begin{array}{c}\text { mean } \\
\log L\end{array}$ & $\begin{array}{c}\text { mean } \\
T_{\text {eff }}\end{array}$ & $\begin{array}{c}\text { mean } \\
Y\end{array}$ \\
\hline \hline NGC 6171 & I & -0.68 & 6 & 0.53 & 1.65 & 7447 & 0.29 \\
M 5 & I & -1.25 & 7 & 0.58 & 1.68 & 7388 & 0.28 \\
M 5 & I & -1.25 & 14 & $0.54 \pm 0.02$ & $1.69 \pm 0.01$ & $7353 \pm 19$ & $0.28 \pm 0.01$ \\
M 3 & I & -1.47 & 5 & 0.59 & 1.71 & 7315 & 0.27 \\
M 9 & II & -1.72 & 1 & 0.60 & 1.72 & 7299 & 0.27 \\
M 55 & II & -1.90 & 5 & 0.53 & 1.75 & 7193 & 0.27 \\
NGC 2298 & II & -1.90 & 2 & 0.59 & 1.75 & 7200 & 0.26 \\
M 68 & II & -2.03 & 16 & 0.70 & 1.79 & 7145 & 0.25 \\
M 15 & II & -2.28 & 6 & 0.73 & 1.80 & 7136 & 0.25 \\
\hline \hline
\end{tabular}

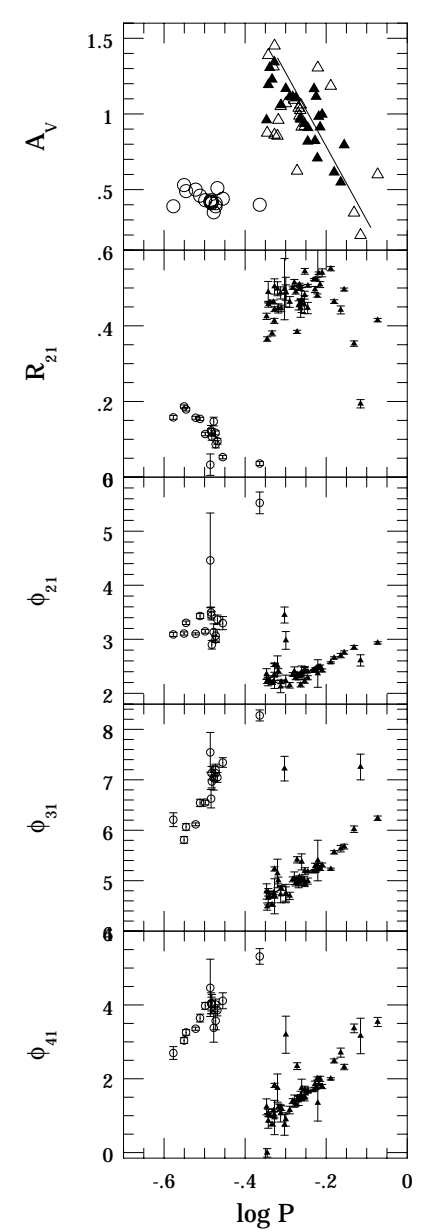

Fig. 3. Amplitude, amplitude ratio and Fourier phase differences as a function of period. RRc variables are plotted with circles, RRab stars with $D_{\mathrm{m}}<3$ with solid triangles and RRab variables with $D_{\mathrm{m}}>3$ with open triangles. The solid line in the upper panel of the figure represents a linear fit to RRab variables in M 3 (Kaluzny et al. 1998)

values of $A_{0}$ (i.e. the mean observed magnitude). The solid lines have a slope of 0.4 and are separated by 0.04 in $\log L$, which represents the one sigma standard deviation in the computed values of luminosity (Simon \& Clement

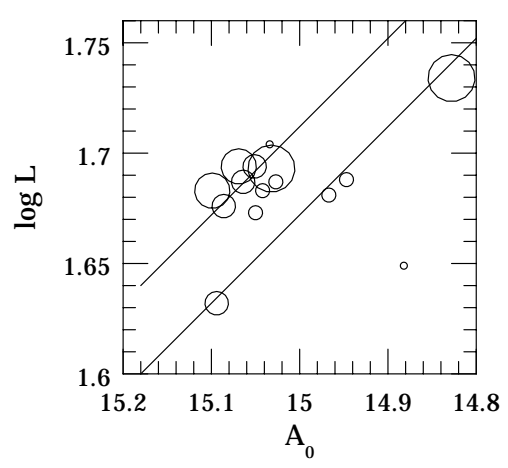

Fig. 4. The dependence between luminosity and visual magnitude for the RRc stars in M 5. The solid lines have a slope of 0.4 and are separated by 0.04 in $\log L$, which represents the uncertainty in the values of $\log L$ computed from $\phi_{31}$ and $P_{1}$. The size of the circles corresponds to the distance from the center of the cluster with larger circles laying at larger distances

1993). Simon and Clement suggested that some of the scatter in this plot for other clusters might be explained by poor photometry resulting from crowding effects in the dense, central regions of the clusters. We have tested this for our sample by plotting in Fig. 4 symbols with size proportional to the distance of a star from the center of the cluster. It can be seen that the most discrepant point is from the center of the cluster (this point is V130 which, as mentioned in the previous section, is badly crowded). The remaining points lay near the solid lines and not between them which may suggest that the problems with crowding do not explain fully the scatter in the $\log L-A_{0}$ plot. The similar situation is in M 55 where Olech et al. (1999a) found that the scatter in the $\log L$ vs. $A_{0}$ relation was not due to crowding effects.

Table 3 presents the mean parameters for RRc stars from several clusters. This table is taken from Kaluzny et al. (1998). A previous determination of the physical parameters of RRc stars in M 5 was made by Clement \& Shelton (1997) who used the data of Reid (1996). Only seven stars from Reid's sample had errors of $\phi_{31}$ smaller than 0.2. Our sample is twice as large, and therefore the derived mean values of mass, luminosity, temperature and 


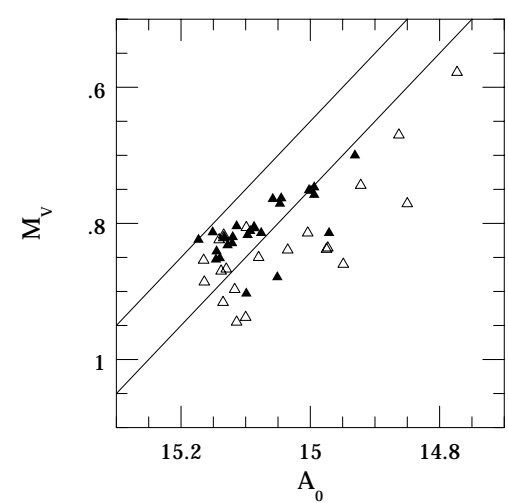

Fig. 5. $M_{V}$ versus mean visual magnitude for the M 5 RRab variables. The envelope lines, plotted with the slope of unity, are separated by $0.1 \mathrm{mag}$ in $M_{V}$, representing the uncertainty in the derived magnitudes (Kovacs \& Jurcsik 1996)

helium abundance are statistically better defined in comparison with the results of Clement \& Shelton (1997).

Clement \& Shelton (1997) noted the existence of correlations between the mean values of several parameters for RRc stars belonging to different clusters. Specifically, an increasing mean mass corresponds to an increasing value of luminosity and to a decreasing value of effective temperature and helium abundance. Our results for M 5 are consistent with these correlations. On the other hand Table 3 contains the recent result of Olech et al. (1999a) who found that the RRc variables in M 55 have a mean mass too small to fit well into the sequence in Table 3.

\subsection{RRab stars}

Several RRab stars exhibit quite a large scatter in their phased light curves. In the case of V63, this is is likely due to poor phase coverage and phasing uncertainty. V2, V4, V8, V14, V27, V65 and V89 display modulation of their light curves which reflects intrinsic variability. The variable V91 is placed in the vicinity of a saturated star and thus its photometry is of poor quality.

The morphology of the light curves is seen to change with period. Two RRab stars with the shortest periods, V4 and V29, reveal broad flat minima and fairly symmetric triangular maxima. Stars with periods of intermediate length have a much steeper rise than decline and their minima are often deformed by bumps. Among long period RRab stars V963 with a small amplitude is rather special. Light curves of other long period stars V43, V75, V9, V87 and V77 all reveal a break in steepness of their rise to the maximum.

Kovacs \& Jurcsik (1996, 1997, and references quoted therein, hereafter KJ) have extended the Fourier analysis of Simon, Teays and Clement to RRab stars. Although their method is still evolving, to retain comparability with earlier results we have used their original formulae as listed by Olech et al. (1999a). Table 4 gives the light curve parameters of M 5 RRab variables in our sample obtained from Fourier fitting. As for the RRc stars, we also list cluster averages and standard deviations of the Fourier parameters as model-free characteristics of the whole cluster.

We have applied the KJ formulae to the values from Table 4. The results are listed in Table 5, which contains values and errors of the absolute magnitude $M_{V}$, metallicity $[\mathrm{Fe} / \mathrm{H}]$, effective temperature $T_{\text {eff }}$. We also list the deviation parameter $D_{\mathrm{m}}$ as calculated from new equations given by Kovacs \& Kanbur (1998). This parameter measures the regularity of the light curve, and according to the original paper of KJ, their equations are valid only for RR Lyr stars with $D_{\mathrm{m}}<3$. A total of 26 stars from our sample satisfy this condition. In the $A_{V}-\log P$ plot presented in Fig. 3 the RRab variables with $D_{\mathrm{m}}<3$ are plotted with solid triangles and these with $D_{\mathrm{m}}>3$ with open triangles. The solid line in this plot represents a linear fit to RRab variables in M 3 (Kaluzny et al. 1998).

Recently Clement \& Shelton (1999) examined the $A_{V}-\log P$ relations for RRab stars in the Oosterhoff type I clusters M 3 and M 107 and Oosterhoff type II clusters M 9 and M 68. They suggested that the $V$ amplitude for a given period is not a function of metal abundance but rather a function of the Oosterhoff type. For example, the clusters M 3 and M 5, which have the same Oosterhoff type, should have identical $A_{V}-\log P$ relations. Inspection of the upper panel of Fig. 3 shows that this is not true. A linear fit to RRab variables in M 3 presented in this figure as a solid line clearly divides the regular RRab stars into two groups. The mean magnitude of the six variables laying above the M 3 fiducial line is $15.00 \pm 0.02$ and the mean magnitude of the regular RRab stars laying under the solid line is $15.10 \pm 0.01$. This strongly suggests that these six RRab stars are in a more advanced evolutionary state than the others. Similar behavior was seen by Kaluzny et al. (1998) among the RRab stars in M 3. After excluding these six stars from our analysis, one can still see that the remaining RRab stars do not fit the relation derived for RRab variables from M 3 but lie below it (toward lower amplitudes and shorter periods). This suggests that the zero point of the linear $A_{V}-\log P$ relation depends on the metallicity of the cluster and not the Oosterhoff type as suggested by Clement \& Shelton (1999).

For 26 RRab stars with $D_{\mathrm{m}}<3$ we obtained the following mean parameters: $M_{V}=0.81,[\mathrm{Fe} / \mathrm{H}]=-1.23$ and $T_{\text {eff }}=6465 \mathrm{~K}$. These values for M 5 fit well into the sequence of physical parameters measured for other clusters with Fourier analysis as summarized in Table 6 .

In Fig. 5 we plot $M_{V}$ values calculated for all our RRab stars against $A_{0}$. Again the RRab variables with $D_{\mathrm{m}}<3$ are plotted with solid triangles and those with $D_{\mathrm{m}}>3$ with open triangles. The solid lines with a slope of unity are separated by $0.1 \mathrm{mag}$, and represent the one sigma uncertainty in the estimation of $M_{V}$ using the KJ formalism. 
Table 4. Light curve parameters for the RRab Lyrae variables in M 5

\begin{tabular}{|c|c|c|c|c|c|c|c|c|c|c|c|c|}
\hline Star & Period & $A_{V}$ & $A_{0}$ & $A_{1}$ & $R_{21}$ & $\sigma_{R_{21}}$ & $\phi_{21}$ & $\sigma_{\phi_{21}}$ & $\phi_{31}$ & $\sigma_{\phi_{31}}$ & $\phi_{41}$ & $\sigma_{\phi_{41}}$ \\
\hline V1 & 0.521777 & 1.11 & 15.145 & 0.366 & 0.500 & 0.003 & 2.351 & 0.033 & 5.026 & 0.049 & 1.394 & 0.067 \\
\hline $\mathrm{V} 2$ & 0.526612 & 1.09 & 15.141 & 0.393 & 0.514 & 0.009 & 2.405 & 0.084 & 5.049 & 0.125 & 1.394 & 0.165 \\
\hline V3 & 0.600149 & 0.71 & 15.076 & 0.254 & 0.480 & 0.004 & 2.463 & 0.019 & 5.311 & 0.030 & 2.007 & 0.044 \\
\hline V4 & 0.449699 & 0.96 & 15.099 & 0.395 & 0.425 & 0.008 & 2.356 & 0.100 & 4.786 & 0.152 & 1.239 & 0.216 \\
\hline V5 & 0.545824 & 1.06 & 15.099 & 0.358 & 0.447 & 0.024 & 2.157 & 0.040 & 4.976 & 0.065 & 1.555 & 0.107 \\
\hline V6 & 0.548891 & 0.92 & 15.138 & 0.301 & 0.468 & 0.033 & 2.397 & 0.109 & 5.373 & 0.159 & 1.748 & 0.241 \\
\hline V8 & 0.546143 & 0.96 & 15.120 & 0.340 & 0.462 & 0.007 & 2.350 & 0.028 & 5.037 & 0.034 & 1.513 & 0.051 \\
\hline V9 & 0.698907 & 0.80 & 14.931 & 0.284 & 0.496 & 0.004 & 2.756 & 0.031 & 5.676 & 0.048 & 2.319 & 0.073 \\
\hline V10 & 0.530662 & 1.10 & 15.133 & 0.365 & 0.490 & 0.006 & 2.290 & 0.037 & 4.952 & 0.057 & 1.338 & 0.079 \\
\hline V11 & 0.595911 & 1.11 & 14.994 & 0.380 & 0.524 & 0.003 & 2.456 & 0.008 & 5.188 & 0.013 & 1.742 & 0.017 \\
\hline V12 & 0.467707 & 1.31 & 15.165 & 0.438 & 0.463 & 0.005 & 2.221 & 0.021 & 4.760 & 0.030 & 1.141 & 0.043 \\
\hline V13 & 0.513002 & 1.11 & 14.971 & 0.376 & 0.463 & 0.014 & 2.148 & 0.051 & 4.700 & 0.071 & 1.153 & 0.103 \\
\hline V14 & 0.487172 & 1.05 & 15.130 & 0.347 & 0.487 & 0.016 & 2.124 & 0.112 & 4.726 & 0.163 & 1.061 & 0.231 \\
\hline V16 & 0.647634 & 1.19 & 14.863 & 0.399 & 0.551 & 0.006 & 2.570 & 0.014 & 5.230 & 0.021 & 1.999 & 0.030 \\
\hline V18 & 0.463956 & 1.23 & 15.151 & 0.484 & 0.380 & 0.007 & 2.191 & 0.024 & 4.527 & 0.035 & 0.771 & 0.052 \\
\hline V19 & 0.469918 & 1.34 & 15.173 & 0.484 & 0.413 & 0.007 & 2.347 & 0.043 & 4.713 & 0.060 & 1.026 & 0.083 \\
\hline V20 & 0.609551 & 0.91 & 15.058 & 0.320 & 0.509 & 0.008 & 2.493 & 0.032 & 5.253 & 0.050 & 1.998 & 0.070 \\
\hline $\mathrm{V} 21$ & 0.604896 & 0.98 & 15.045 & 0.330 & 0.539 & 0.003 & 2.490 & 0.013 & 5.229 & 0.022 & 1.844 & 0.031 \\
\hline V24 & 0.478471 & 0.85 & 15.100 & 0.315 & 0.498 & 0.018 & 2.507 & 0.186 & 5.143 & 0.283 & 1.748 & 0.382 \\
\hline $\mathrm{V} 27$ & 0.470532 & 1.45 & 15.004 & 0.498 & 0.504 & 0.020 & 2.327 & 0.236 & 4.691 & 0.347 & 0.951 & 0.465 \\
\hline V28 & 0.543865 & 0.97 & 15.121 & 0.328 & 0.500 & 0.007 & 2.348 & 0.016 & 5.035 & 0.022 & 1.463 & 0.030 \\
\hline V29 & 0.451332 & 0.88 & 15.164 & 0.359 & 0.365 & 0.006 & 2.231 & 0.049 & 4.487 & 0.072 & -0.008 & 0.116 \\
\hline V30 & 0.592207 & 0.82 & 15.093 & 0.278 & 0.496 & 0.008 & 2.429 & 0.042 & 5.284 & 0.063 & 1.854 & 0.083 \\
\hline V32 & 0.457797 & 1.31 & 15.146 & 0.452 & 0.462 & 0.002 & 2.233 & 0.006 & 4.680 & 0.009 & 0.997 & 0.014 \\
\hline V33 & 0.501575 & 1.17 & 15.128 & 0.380 & 0.489 & 0.039 & 2.228 & 0.109 & 4.736 & 0.142 & 0.898 & 0.181 \\
\hline V34 & 0.568119 & 0.82 & 15.087 & 0.293 & 0.447 & 0.015 & 2.288 & 0.038 & 4.992 & 0.060 & 1.682 & 0.072 \\
\hline V38 & 0.470437 & 0.86 & 15.114 & 0.342 & 0.444 & 0.006 & 2.522 & 0.026 & 5.232 & 0.042 & 1.820 & 0.058 \\
\hline V39 & 0.589035 & 1.17 & 14.999 & 0.389 & 0.522 & 0.003 & 2.431 & 0.009 & 5.181 & 0.013 & 1.687 & 0.018 \\
\hline V41 & 0.488577 & 1.06 & 15.140 & 0.404 & 0.446 & 0.009 & 2.213 & 0.035 & 4.851 & 0.047 & 1.202 & 0.062 \\
\hline V43 & 0.660177 & 0.61 & 15.047 & 0.224 & 0.464 & 0.005 & 2.656 & 0.021 & 5.563 & 0.033 & 2.484 & 0.056 \\
\hline V45 & 0.616632 & 1.00 & 14.994 & 0.324 & 0.540 & 0.010 & 2.423 & 0.028 & 5.310 & 0.041 & 1.790 & 0.057 \\
\hline V47 & 0.539739 & 1.04 & 15.134 & 0.348 & 0.454 & 0.012 & 2.316 & 0.067 & 4.960 & 0.100 & 1.409 & 0.132 \\
\hline V52 & 0.501785 & 1.07 & 14.973 & 0.373 & 0.488 & 0.020 & 2.979 & 0.164 & 4.745 & 0.182 & 3.194 & 0.502 \\
\hline V54 & 0.454239 & 1.19 & 15.051 & 0.427 & 0.457 & 0.008 & 2.250 & 0.050 & 4.770 & 0.074 & 1.072 & 0.100 \\
\hline V56 & 0.534849 & 0.62 & 15.135 & 0.255 & 0.384 & 0.004 & 2.353 & 0.040 & 5.414 & 0.062 & 2.342 & 0.092 \\
\hline V59 & 0.542027 & 0.99 & 14.975 & 0.324 & 0.509 & 0.004 & 2.339 & 0.014 & 5.076 & 0.021 & 1.486 & 0.030 \\
\hline V61 & 0.568647 & 0.91 & 15.097 & 0.312 & 0.506 & 0.004 & 2.402 & 0.011 & 5.179 & 0.016 & 1.662 & 0.021 \\
\hline V63 & 0.497993 & 0.70 & 15.263 & 0.565 & 0.497 & 0.081 & 3.449 & 0.148 & 7.221 & 0.244 & 0.754 & 0.284 \\
\hline V64 & 0.544492 & 1.02 & 15.134 & 0.337 & 0.504 & 0.003 & 2.336 & 0.017 & 5.009 & 0.025 & 1.445 & 0.037 \\
\hline V65 & 0.480758 & 0.96 & 15.117 & 0.363 & 0.446 & 0.012 & 2.409 & 0.047 & 5.004 & 0.067 & 1.219 & 0.098 \\
\hline V74 & 0.453987 & 1.39 & 15.080 & 0.464 & 0.489 & 0.028 & 2.233 & 0.093 & 4.657 & 0.136 & 0.859 & 0.199 \\
\hline V75 & 0.685471 & 0.55 & 15.002 & 0.208 & 0.442 & 0.010 & 2.698 & 0.041 & 5.635 & 0.065 & 2.708 & 0.123 \\
\hline V77 & 0.845261 & 0.60 & 14.773 & 0.234 & 0.415 & 0.005 & 2.934 & 0.021 & 6.238 & 0.044 & 3.539 & 0.123 \\
\hline V81 & 0.557292 & 0.94 & 15.087 & 0.331 & 0.456 & 0.024 & 2.420 & 0.061 & 4.999 & 0.089 & 1.526 & 0.052 \\
\hline V82 & 0.558927 & 0.92 & 15.035 & 0.298 & 0.544 & 0.008 & 2.446 & 0.046 & 5.192 & 0.065 & 1.710 & 0.088 \\
\hline V87 & 0.739210 & 0.35 & 14.922 & 0.150 & 0.353 & 0.007 & 2.850 & 0.036 & 6.021 & 0.064 & 3.365 & 0.118 \\
\hline V89 & 0.558454 & 0.94 & 15.114 & 0.316 & 0.481 & 0.010 & 2.224 & 0.038 & 4.937 & 0.054 & 1.481 & 0.080 \\
\hline V91 & 0.601589 & 1.31 & 14.850 & 0.361 & 0.640 & 0.111 & 2.367 & 0.255 & 5.400 & 0.402 & 1.344 & 0.490 \\
\hline V963 & 0.766991 & 0.20 & 14.949 & 0.093 & 0.194 & 0.011 & 2.609 & 0.104 & 7.254 & 0.255 & 3.160 & 0.481 \\
\hline Mean & 0.554059 & 0.97 & 15.066 & 0.346 & 0.470 & - & 2.429 & - & 5.172 & - & 1.634 & - \\
\hline
\end{tabular}


Table 5. Physical parameters of the RRab variables in M 5

\begin{tabular}{|c|c|c|c|c|c|c|}
\hline Star & $M_{V}$ & $\sigma_{M_{V}}$ & {$[\mathrm{Fe} / \mathrm{H}]$} & $\sigma_{[\mathrm{Fe} / \mathrm{H}]}$ & $T_{\text {eff }}$ & $D_{\mathrm{m}}$ \\
\hline$\overline{\overline{\text { V1 }}}$ & $\overline{0.841}$ & 0.085 & $\overline{-1.092}$ & 0.068 & $\overline{c 6542}$ & $\overline{1.38}$ \\
\hline $\mathrm{V} 2$ & 0.824 & 0.086 & -1.087 & 0.168 & 6553 & 3.35 \\
\hline V3 & 0.814 & 0.093 & -1.133 & 0.045 & 6377 & 1.09 \\
\hline V4 & 0.903 & 0.081 & -1.026 & 0.206 & 6626 & 2.67 \\
\hline V5 & 0.806 & 0.085 & -1.289 & 0.089 & 6461 & 3.31 \\
\hline V6 & 0.870 & 0.094 & -0.772 & 0.215 & 6539 & 4.13 \\
\hline V8 & 0.820 & 0.086 & -1.210 & 0.049 & 6476 & 0.92 \\
\hline V9 & 0.700 & 0.101 & -1.174 & 0.076 & 6298 & 2.49 \\
\hline V10 & 0.821 & 0.084 & -1.241 & 0.078 & 6506 & 0.79 \\
\hline V11 & 0.747 & 0.089 & -1.274 & 0.026 & 6429 & 2.31 \\
\hline V12 & 0.854 & 0.078 & -1.159 & 0.049 & 6614 & 13.93 \\
\hline V13 & 0.814 & 0.080 & -1.483 & 0.100 & 6481 & 1.84 \\
\hline V14 & 0.867 & 0.082 & -1.309 & 0.221 & 6533 & 7.47 \\
\hline V16 & 0.670 & 0.091 & -1.497 & 0.037 & 6332 & 4.75 \\
\hline V18 & 0.813 & 0.074 & -1.452 & 0.061 & 6604 & 2.96 \\
\hline V19 & 0.824 & 0.077 & -1.234 & 0.086 & 6628 & 2.77 \\
\hline V20 & 0.764 & 0.092 & -1.261 & 0.071 & 6372 & 2.44 \\
\hline V21 & 0.763 & 0.091 & -1.267 & 0.036 & 6393 & 1.04 \\
\hline V24 & 0.938 & 0.092 & -0.701 & 0.381 & 6600 & 4.27 \\
\hline V 27 & 0.814 & 0.084 & -1.267 & 0.468 & 6634 & 3.67 \\
\hline V28 & 0.829 & 0.086 & -1.200 & 0.034 & 6480 & 1.32 \\
\hline V29 & 0.886 & 0.076 & -1.438 & 0.105 & 6638 & 6.83 \\
\hline V30 & 0.811 & 0.092 & -1.126 & 0.087 & 6409 & 0.75 \\
\hline V32 & 0.853 & 0.077 & -1.213 & 0.033 & 6628 & 1.11 \\
\hline V33 & 0.832 & 0.081 & -1.373 & 0.192 & 6542 & 1.69 \\
\hline V34 & 0.807 & 0.087 & -1.388 & 0.083 & 6384 & 2.28 \\
\hline V38 & 0.945 & 0.088 & -0.538 & 0.060 & 6650 & 3.21 \\
\hline V39 & 0.752 & 0.089 & -1.247 & 0.026 & 6449 & 2.65 \\
\hline V41 & 0.851 & 0.081 & -1.148 & 0.067 & 6583 & 1.71 \\
\hline V43 & 0.771 & 0.099 & -1.116 & 0.055 & 6289 & 1.24 \\
\hline V45 & 0.758 & 0.093 & -1.223 & 0.060 & 6401 & 1.62 \\
\hline V47 & 0.817 & 0.085 & -1.279 & 0.135 & 6477 & 14.53 \\
\hline V52 & 0.836 & 0.082 & -1.362 & 0.247 & 6307 & 35.10 \\
\hline V54 & 0.879 & 0.079 & -1.073 & 0.102 & 6645 & 1.46 \\
\hline V56 & 0.916 & 0.093 & -0.642 & 0.087 & 6495 & 13.62 \\
\hline V59 & 0.838 & 0.087 & -1.135 & 0.033 & 6492 & 14.87 \\
\hline V61 & 0.817 & 0.089 & -1.139 & 0.028 & 6453 & 0.71 \\
\hline V64 & 0.821 & 0.086 & -1.238 & 0.039 & 6476 & 14.83 \\
\hline V65 & 0.897 & 0.084 & -0.900 & 0.091 & 6628 & 3.96 \\
\hline V74 & 0.850 & 0.077 & -1.223 & 0.186 & 6647 & 4.21 \\
\hline V75 & 0.751 & 0.101 & -1.156 & 0.096 & 6237 & 2.16 \\
\hline V77 & 0.578 & 0.115 & -1.208 & 0.095 & 6075 & 4.94 \\
\hline V81 & 0.805 & 0.087 & -1.320 & 0.122 & 6439 & 2.87 \\
\hline V82 & 0.839 & 0.090 & -1.069 & 0.089 & 6464 & 3.52 \\
\hline V87 & 0.744 & 0.109 & -0.927 & 0.104 & 6173 & 3.64 \\
\hline V89 & 0.804 & 0.086 & -1.410 & 0.076 & 6414 & 2.25 \\
\hline V91 & 0.771 & 0.103 & -1.019 & 0.541 & 6520 & 8.83 \\
\hline V963 & 0.860 & 0.133 & 0.581 & 0.366 & 6510 & 24.71 \\
\hline
\end{tabular}

There are three stars (V4, V13, V54) among the RRab variables with regular light curves (i.e. with $D_{\mathrm{m}}<3$ ) which are about 0.05 mag too bright for their absolute magnitude and do not lay between the envelope lines in Fig. 5. These objects are also the stars with $D_{\mathrm{m}}<3$ that are closest to the center of the cluster and their true

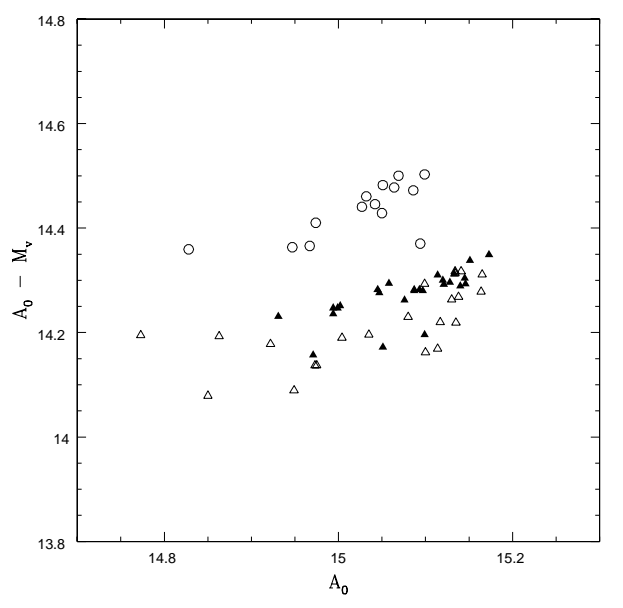

Fig. 6. Apparent distance modulus plotted against $A_{0}$ for $\mathrm{RRc}$ stars (open circles), RRab stars with $D_{\mathrm{m}}<3.0$ (closed triangles), and RRab stars with $D_{\mathrm{m}}>3.0$ (open triangles)

visual magnitudes may be slightly fainter than the values derived from our data.

The range of temperature for the RRc and RRab stars is consistent with that presented by Reid (1996) who calculated temperatures both from pulsation theory (Carney et al. 1992) and from $(V-I)$ colors.

\subsection{The distance modulus to $M 5$}

In Fig. 6 we plot the apparent distance moduli of the RRab and RRc stars in our sample as measured by the Fourier technique. For the RRab stars $M_{V}$ is taken from Table 5. For the RRc stars, $M_{V}$ has been calculated from $\log L$ (Table 2), with $M_{V}=M_{\mathrm{BOL}}-\mathrm{BC}$, and taking $\mathrm{BC}=$ $0.06[\mathrm{Fe} / \mathrm{H}]+0.06$ (Sandage \& Cacciari 1990). We adopt $[\mathrm{Fe} / \mathrm{H}]=-1.23$ (see Table 6 ).

The different zero point calibrations of the RRc stars by $\mathrm{SC}$ and the RRab stars by JK is clear from Fig. 6 . The zero points of the RRc stars are calibrated directly from the hydrodynamical models, while the RRab stars are calibrated by Baade-Wesselink observations of field RR Lyr stars. The mean apparent distance modulus as measured from the RRc stars (dropping V76 and V78, see discussion in Sect. 2.1.1) is $(m-M)_{V}=14.47 \pm 0.11$. For the RRab stars with $D_{\mathrm{m}}<3.0$ the mean apparent distance modulus is $(m-M)_{V}=14.27 \pm 0.04$. While the discrepancy arising from the zero point calibration of the Fourier technique is substantial, the distance modulus obtained for RRab stars is consistent with the value of $(m-M)_{V}=14.33$ (Harris 1996). On the other hand recent determinations of distance modulus to M 5 give the values between 14.41 and 14.58 (Sandquist et al. 1996; Reid 1998). The distance modulus derived by us from RRc stars agrees within the errors with these recent determinations. It indicates that hydrodynamical models of Simon \& Clement (1993) give the correct luminosities of RRc stars. 
Table 6. Mean parameters for cluster RRab stars based on the method of KJ

\begin{tabular}{|l|c|c|c|c|c|c|c|}
\hline \hline Cluster & $\begin{array}{c}\text { No. of } \\
\text { stars }\end{array}$ & $\begin{array}{c}\text { mean } \\
P\end{array}$ & $\begin{array}{c}\text { mean } \\
\phi_{31}\end{array}$ & $\begin{array}{c}\text { mean } \\
M_{V}\end{array}$ & $\begin{array}{c}\text { mean } \\
{[\mathrm{Fe} / \mathrm{H}]}\end{array}$ & $\begin{array}{c}\text { mean } \\
T_{\text {eff }}\end{array}$ & Reference \\
\hline \hline NGC 6171 & 3 & 0.536 & $5.22 \pm 0.18$ & $0.85 \pm 0.02$ & $-0.91 \pm 0.13$ & $6619 \pm 64$ & Clement \& Shelton (1997) \\
M 5 & 26 & 0.555 & $5.05 \pm 0.06$ & $0.81 \pm 0.01$ & $-1.23 \pm 0.03$ & $6465 \pm 22$ & this work \\
NGC 1851 & 7 & 0.555 & $5.10 \pm 0.06$ & $0.80 \pm 0.01$ & $-1.17 \pm 0.03$ & $6494 \pm 35$ & Walker (1999) \\
M 3 & 17 & 0.562 & $4.95 \pm 0.05$ & $0.78 \pm 0.01$ & $-1.42 \pm 0.03$ & $6438 \pm 18$ & Kaluzny et al. (1998) \\
M 55 & 3 & 0.655 & $5.21 \pm 0.22$ & $0.68 \pm 0.02$ & $-1.56 \pm 0.16$ & $6325 \pm 69$ & Olech et al. (1999a) \\
\hline \hline
\end{tabular}

It is interesting to note that the apparent distance moduli for individual stars in Fig. 6 appears to be correlated with $A_{0}$ for both the RRab and RRc star samples. Scale errors in $A_{0}$ are not likely to be the explanation since the range in $A_{0}$ is small. In addition, while there does seem to be a similar correlation shown in the comparison with Reid's (1996) photometry for the small sample of RRc stars in common between the two studies (see Fig. 2), no such correlation is seen for the RRab stars. The slope of these correlations is substantial, and could compromise attempts to use the Fourier analysis of RR Lyr light curves to derive distances to globular clusters. Accurate CCD photometry of samples of cluster RR Lyrae stars is needed to address this issue.

\section{Conclusions}

We have presented accurate $V$-band CCD photometry of $65 \mathrm{RR}$ Lyr variables in the globular cluster M 5. Among these stars we have detected 49 fundamental mode pulsators, 15 1st overtone pulsators and one possible 2nd overtone pulsator.

Four of our RRc stars with shortest periods show remarkably similar light curves with a clear bump visible just before the maxima and increased scatter of the observational points around maximum light. We suggest that these features, due to the short pulsation periods of the stars, are caused by interaction between the first and the second overtones of radial pulsations.

Our precise photometry allowed us to obtain accurate Fourier coefficients for $14 \mathrm{RRc}$ stars (twice as many as in previous investigations) and to estimate the physical parameters of these objects. We have measured the mean mass of these stars to be $0.54 \mathcal{M}_{\odot}$, the mean logarithm of luminosity $L / L_{\odot}$ equal to 1.69 , the mean effective temperature of $7353 \mathrm{~K}$ and the mean relative helium abundance equal to 0.28 . These values are consistent with the previous determinations of physical parameters of M 5 and also with recent theoretical models (Reid 1996; Clement \& Shelton 1997; Sandquist et al. 1996; Caputo et al. 1999).

As many as 26 out of the 49 observed RRab stars have regular light curves as measured by the $D_{\mathrm{m}}$ parameter of KJ and thus are suitable for estimating the absolute magnitude, metallicity and effective temperature from the Fourier coefficients of these stars. From this sample we obtained $M_{V}=0.81 \mathrm{mag},[\mathrm{Fe} / \mathrm{H}]=-1.23$ and $T_{\text {eff }}=6465 \mathrm{~K}$. The derived metallicity corresponds to the Jurcsik (1995) scale which was used by KJ for calibrating their method. Jurcsik (1995) gave $[\mathrm{Fe} / \mathrm{H}]=-1.25$ for $\mathrm{M} 5$ on her scale and this value is in excellent agreement with our estimate.

We also found that six of the regular RRab variables are in a more advanced evolutionary state than the rest of our sample. A detailed inspection of the $A_{V}-\log P$ relations for the RRab stars from M 3 and M 5 suggests that the $V$ amplitude of RRab variables at a given period is a function of the metallicity rather than the Oosterhoff type of the cluster.

We estimate distance moduli of $(m-M)_{V}=14.47$ for the RRc stars in our sample, and $(m-M)_{V}=14.27$ for the RRab stars. A possible correlation between measured luminosity as derived from the Fourier coefficients and apparent brightness was identified for both the RRc and RRab stars in our sample.

Acknowledgements. JK, AO and WK were supported by the Polish Committee of Scientific Research through grant 2P03D011-12 and by NSF grant AST-9528096 to Bohdan Paczyński. ASC acknowledges support by KBN grant 2P03C-001-12 and WP was supported by KBN grant 2P03-010-15.

\section{References}

Butler D., 1975, ApJ 200, 68

Caputo F., Castellani V., Marconi M., Ripepi V., 1999, MNRAS 306, 815

Carney B.W., Storm J., Jones R., 1992, ApJ 386, 663

Carretta E., Gratton R.G., 1997, A\&AS 121, 95

Clement C.M., Janulak M., Simon N.R., 1992, ApJ 395, 192

Clement C.M., 1997, An Update to Helen Sawyer-Hogg's Third Catalogue of Variable Stars in Globular Clusters (private communication)

Clement C.M., Shelton I., 1997, AJ 113, 1711

Clement C.M., Shelton I., 1999, ApJL 515, L85

Drissen L., Shara M.M., 1998, AJ 115, 725

Harris W.E., 1996, AJ 112, 1487

Jurcsik J., 1995, Acta Astron. 45, 653

Kaluzny J., Hilditch R.W., Clement C., Rucinski S.M., 1998, MNRAS 296, 347

Kaluzny J., Thompson I., Krzeminski W., Pych W., 1999 (submitted to A\&A) 
Kovacs G., Jurcsik J., 1996, ApJ 466, L17

Kovacs G., Jurcsik J., 1997, A\&A 322, 218

Kovacs G., Kanbur S.M., 1998, MNRAS 295, 834

Minniti D., Alcock C., Alves D.R., Allsman R.A., Axelrod T.S., et al., 1997, Proceeding of the 12th IAP Colloquium Variable Stars and the Astrophysical Returns of Microlensing Surveys

Olech A., 1997, Acta Actron. 47, 183

Olech A., Kaluzny J., Thompson I., et al., 1999a, AJ 118, 442

Olech A., Woźniak P.R., Alard C., Kaluzny J., Thompson I.B., 1999b, MNRAS 310, 759

Reid I.N., 1996, MNRAS 278, 367

Reid I.N., 1998, AJ 115, 204

Sandage A., 1981, ApJ 248, 161

Sandage A., Cacciari C., 1990, ApJ 350, 645
Sandquist E., Bolte M., Stetson P., Hesser J.E., 1996, ApJ 470, 910

Sawyer Hogg H., 1973, Publ. David Dunlap Obs. 3, No. 6

Schwarzenberg-Czerny A., 1997, ApJ 489, 941

Schwarzenberg-Czerny A., Kaluzny J., 1998, MNRAS 300, 251

Simon N.R., Teays T.J., 1982, ApJ 261, 586

Simon N.R., 1989, ApJL 343, L17

Simon N.R., Clement C.M., 1993, ApJ 410, 526

Stellingwerf R.F., Gautschy A., Dickens R.J., 1987, ApJ 313, L75

Walker A.R., Nemec J.M., 1996, AJ 112, 2026

Walker A.R., 1999, AJ 116, 220

Yan L., Reid I.N., 1996, MNRAS 279, 751

Zhukov L.V., 1971, Trudy Pulkovo Obs. Ser. 2, 78, 160

Zinn R., 1985, ApJ 293, 424 